OPEN ACCESS

Edited by:

Barbara Fazekas De St Groth,

Centenary Institute of Cancer Medicine and Cell Biology, Australia

Reviewed by: Toshinori Nakayama,

Chiba University, Japan Karin Schilbach,

Children's University Hospital

Tübingen, Germany

*Correspondence:

Vassiliki A. Boussiotis

vboussio@bidmc.harvard.edu

Specialty section:

This article was submitted to

T Cell Biology,

a section of the journal

Frontiers in Immunology

Received: 11 February 2016

Accepted: 18 April 2016

Published: 03 May 2016

Citation:

Karantanos T, Christofides A, Bardhan K, Li L and Boussiotis VA

(2016) Regulation of T Cell Differentiation and Function by EZH2.

Front. Immunol. 7:172.

doi: 10.3389/fimmu.2016.00172

\section{Regulation of T Cell Differentiation and Function by EZH2}

\author{
Theodoros Karantanos ${ }^{1,2,3}$, Anthos Christofides ${ }^{1,2}$, Kankana Bardhan ${ }^{1,2}$, Lequn Li $^{1,2}$ and \\ Vassiliki A. Boussiotis ${ }^{1,2,4 *}$
}

${ }^{1}$ Division of Hematology-Oncology, Beth Israel Deaconess Medical Center, Harvard Medical School, Boston, MA, USA, ${ }^{2}$ Department of Medicine, Beth Israel Deaconess Medical Center, Harvard Medical School, Boston, MA, USA, ${ }^{3}$ General Internal Medicine Section, Boston Medical Center, Boston University School of Medicine, Boston, MA, USA, ${ }^{4}$ Beth Israel Deaconess Cancer Center, Harvard Medical School, Boston, MA, USA

The enhancer of zeste homolog 2 (EZH2), one of the polycomb-group proteins, is the catalytic subunit of Polycomb-repressive complex 2 (PRC2) and induces the trimethylation of the histone $\mathrm{H} 3$ lysine 27 (H3K27me3) promoting epigenetic gene silencing. EZH2 contains a SET domain promoting the methyltransferase activity, while the three other protein components of PRC2, namely EED, SUZ12, and RpAp46/48, induce compaction of the chromatin permitting EZH2 enzymatic activity. Numerous studies highlight the role of this evolutionary conserved protein as a master regulator of differentiation in humans involved in the repression of the homeotic gene and the inactivation of $\mathrm{X}$-chromosome. Through its effects in the epigenetic regulation of critical genes, EZH2 has been strongly linked to cell cycle progression, stem cell pluripotency, and cancer biology, being currently at the cutting edge of research. Most recently, EZH2 has been associated with hematopoietic stem cell proliferation and differentiation, thymopoiesis and lymphopoiesis. Several studies have evaluated the role of EZH2 in the regulation of $T$ cell differentiation and plasticity as well as its implications in the development of autoimmune diseases and graft-versus-host disease (GVHD). The aim of this review is to summarize the current knowledge regarding the role of EZH2 in the regulation of the differentiation and function of $T$ cells focusing on possible applications in various immune-mediated conditions, including autoimmune disorders and GVHD.

Keywords: T cells, $\mathrm{T}$ cell differentiation, $\mathrm{T}$ cell activation, tumor immunity, EZH2

\section{INTRODUCTION}

The enhancer of zeste homolog 2 (EZH2), one of the polycomb-group (PcG) proteins, is the catalytic subunit of Polycomb-repressive complex 2 (PRC2) and induces the trimethylation of the histone $\mathrm{H} 3$ lysine 27 (H3K27me3) promoting epigenetic gene silencing $(1,2)$. EZH2 contains a SET domain promoting the methyltransferase activity, while the three other protein components of $\mathrm{PRC} 2$, namely EED, SUZ12, and RpAp46/48, induce compaction of the chromatin permitting the enzymatic activity of EZH2 (3). Numerous studies highlight the role of this evolutionary conserved protein as a master regulator of differentiation in humans involved in the repression of the homeotic (Hox) gene and the inactivation of $\mathrm{X}$-chromosome (3-6). 
Through its implications in the epigenetic regulation of critical genes, EZH2 has been strongly linked to cell cycle progression (7), stem cell pluripotency $(8,9)$, and cancer biology being currently at the cutting edge of research. At the molecular level, maintaining proper levels of EZH2 is critical for the normal function of cells, while its aberrant expression has been implicated in the induction of cell proliferation and oncogenesis $(10,11)$. Interestingly, EZH2 is aberrantly overexpressed in a variety of neoplasms compared to normal tissues $(11,12)$ and is currently the subject of intense research for the better understanding of cancer biology. Thus, EZH2 is currently evaluated as a new biomarker and a novel target for cancer therapy.

Most recently, EZH2 has been associated with hematopoietic stem cell proliferation and differentiation $(13,14)$, thymopoiesis (14), and lymphopoiesis (15). Numerous studies have evaluated the role of $\mathrm{EZH} 2$ in the regulation of $\mathrm{T}$ cell differentiation and plasticity as well as its implications in the development of autoimmune diseases and graft-versus-host disease (GVHD). Given the currently evolving development of EZH2 inhibitors for human malignancies, the introduction of these inhibitors as modulators of immune-mediated diseases and activation of the immune system in patients with cancers is a promising opportunity.

\section{EZH2 AND REGULATION OF T CELL DIFFERENTIATION}

\section{T Cell Lineage Commitment}

The exposure of naïve $\mathrm{T}$ helper (Th) cells to an antigen in the context of distinct extracellular microenvironment queues leads to differentiation into the effector lineages namely Th1, Th2, and Th17 expressing distinct cytokines genes $(16,17)$. It is known that Th1 lineage produces IFN- $\gamma$ and is implicated in anti-microbial and anti-viral immunity, while Th2 cells produce IL- 4 and are involved in immunity against parasites as well as in the pathogenesis of allergic reactions through the activation of $B$ cells and the subsequent production of $\operatorname{IgE}$ antibodies inducing the activity of mast cells (18). Th17 cells produce mainly IL-17 and IL-22 and are critical for the protection against certain extracellular pathogens, such as Candida albicans and Staphylococcus aureus, but are also involved in the pathophysiology of autoimmune conditions, such as inflammatory bowel disease $(19,20)$. The differentiation of naive Th cells is promoted by a variety of cytokines produced in the setting of localized or systemic inflammation and is associated with a pattern of expression of lineage-specific transcription factors. Particularly, IL-12 promotes the polarization toward Th1 lineage associated with the induction of the T-bet transcriptional factor, while IL-4 potentiates the differentiation toward Th2 lineage that is connected with induction of the GATA-3 transcription factor (21). For the differentiation of Th17 cells, TGF-beta, IL-6, and activation of STAT3 and ROR $\gamma$ transcription factors have indispensable roles (22).

The expression of the transcription factor forkhead box P3 (FOXP3) defines a subset of CD $4^{+}$cells with inhibitory functions called $\mathrm{T}$ regulatory cells (Tregs). Natural Tregs differentiate in the thymus maintaining and expanding the FOXP3 expression with the involvement of IFN- $\gamma$, TGF-beta, and IL-2, while naive $\mathrm{FOXP}^{-}{ }^{-}$cells can differentiate directly in the periphery to
$\mathrm{FOXP}^{+}$de novo induced Tregs through the exposure to antigens with the involvement mainly of TGF-beta. Natural Tregs expressing T-bet transcription factor are known to suppress the function and expansion of Th1 cells, while Treg expressing IRF4 and Stat 3 are critical for the inhibition of Th2 and Th17 cells, respectively (23). These conclusions highlight the importance of Tregs development and maintenance, which is controlled by numerous factors, including cytokines, transcription factors, and epigenetic regulators.

\section{EZH2 and T Cell Differentiation}

Recent studies highlight the role of EZH2 in the differentiation and plasticity of $\mathrm{T}$ cells and introduce a new critical player with possible therapeutic implications. Data presented in an early study by Raaphorst et al. supported that the expression of the PcG proteins BMI-1 and EZH2 in mature peripheral $\mathrm{T}$ cells is mutually exclusive and associated with their proliferation status (24). Of note, particular patterns of EZH2 and BMI-1 expression characterize different stages of $\mathrm{T}$ cells differentiation in the thymus, suggesting a critical regulatory role of PcG proteins in lymphopoiesis through the stabilization of gene expression patterns that will lead to a lineage choice (24).

Subsequent studies using EZH2-deficient T cells determined that EZH2 has a key role in the differentiation and plasticity of Th1 and Th2 T cells and a mandatory role on the survival of T effector cells. Specifically, Zhang et al. evaluated the role of EZH2 expression in the differentiation of naïve $\mathrm{CD} 4^{+} \mathrm{T}$ cells and the survival of effector cells, using a model of transgenic $\mathrm{EZH} 2{ }^{\mathrm{fl} / \mathrm{fl}} \mathrm{CD} 4$-Cre mice for T cell-specific EZH2 deletion (25). These studies revealed that the proportion of effector Th0, Th1, Th2, and Th17 but not iTreg cells in the $\mathrm{EZH} 2{ }^{\mathrm{fl} / \mathrm{fl}} \mathrm{CD} 4$-Cre mice were decreased by day 6 after birth (25). Interestingly, the study showed that EZH2 deletion was associated with upregulation of IFN- $\gamma$ and IL-10 under Th0 conditions, enhanced induction of IFN- $\gamma$ under Th1 conditions and increased expression of IL-10 under Th2 conditions (25). Similarly, Yang et al. showed that EZH2-deficient $\mathrm{CD}^{+} \mathrm{T}$ cells produce higher amounts of IFN- $\gamma$, IL-13, and IL-17 in Th1, Th2, and Th17 conditions, respectively, compared to control T cells, suggesting that EZH2 suppresses the expression of lineage signature cytokines (26). Together these studies indicate that EZH2 has an inhibitory role in the Th1 and Th2 differentiation (Figure 1A). These conclusions are consistent with the results of Tumes et al. who showed that EZH2-deficient Th cells produce more IFN- $\gamma$ during stimulation in the presence of IL-12, which induces Th1 polarization and more IL-4, IL-5, and IL-13 during stimulation in the presence of IL-4, which induces Th2 polarization (27). Moreover, in the same study was found that EZH2deficient T cells express higher amounts of T-bet and Gata-3 (27), critical transcriptional factors for the Th1 and Th2 differentiation, respectively. Of note, Tumes et al. showed that, compared to WT cells, Th1 polarized EZH2-deficient T cells express higher levels of IL-4, IL-5, and IL-13 when exposed to Th2 conditions and Th2 polarized EZH2-deficient T cells express higher levels of IFN- $\gamma$ when exposed to Th1 conditions (27), suggesting that EZH2-deficient cells demonstrate increased plasticity. The inhibitory role of EZH2 on Th2 differentiation was further confirmed by the finding that the transfer of oval-albumin (OVA)-specific 


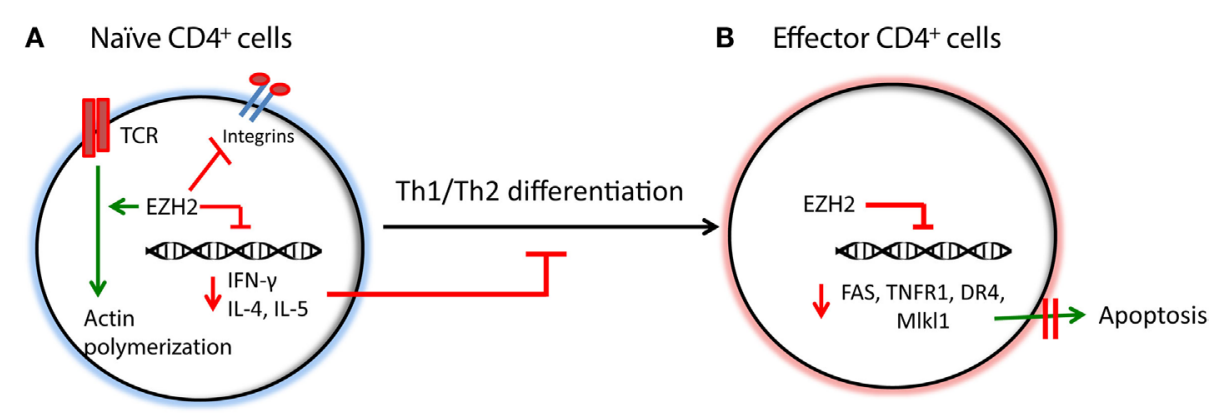

C Regulatory T cells

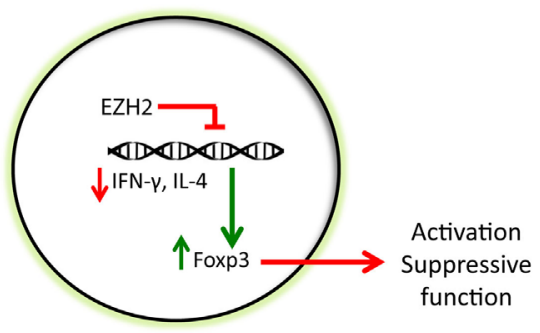

Cancer cells

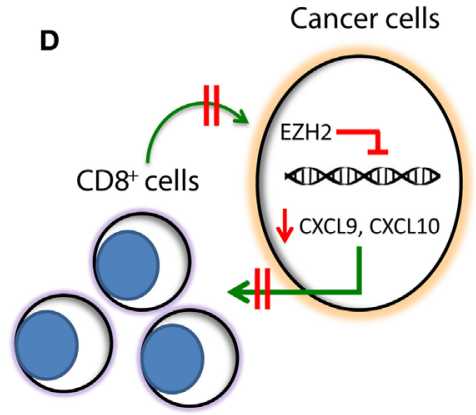

FIGURE 1 | Summary of the functions of EZH2 in T cells, regulatory T cells, and cancer-mediated immune regulation. (A) EZH2 downregulates the expression of Th1/Th2 cytokines, such as IFN- $\gamma$ and IL-4/IL-5, respectively, inhibiting the Th1/Th2 differentiation of naïve CD4+ T cells. EZH2 inhibits integrindependent migration of $\mathrm{T}$ cells while promoting TCR-dependent actin polymerization. (B) In effector CD4 ${ }^{+} \mathrm{T}$ cells EZH2 inhibits the expression of apoptotic molecules, such as FAS, TNFR1, DR4, and Mlkl1, promoting their survival and sustaining the immune responses. (C) EZH2 downregulates the expression of IFN- $\gamma$ and IL-4, while promoting the expression of Foxp3 and the activation of T regulatory cells. (D) In cancer cells, EZH2 inhibits the expression of Th1 chemokines, such as CXCL9 and CXCL10 thereby compromising the trafficking of CD8 ${ }^{+} \mathrm{T}$ cells, which mediate cytotoxic effects on cancer cells and promote anti-tumor function.

EZH2-deficient Th2 cells in C57BL/6 mice was associated with the development of exaggerated allergic asthma characterized by increased eosinophilic inflammation and increased proportion of effector memory $\mathrm{CD} 4^{+} \mathrm{T}$ cells, which produced increased amounts of IL-4 and IL-5 compared to the transfer of WT Th2 cells (27). These findings provided compelling evidence that EZH2 inhibits the differentiation and plasticity of naive T cells. Based on these observations, it can be hypothesized that EZH2 inhibitors might enhance immune responses. In this context, EZH2 inhibitors might promote IFN- $\gamma$-producing T cells and can be used in order to enhance anti-tumor immunity, while EZH2 activating agents might serve as promising therapies for the suppression of Th1- and Th2-dependent autoimmune diseases.

It should be noted that the results of the various groups regarding the role of $\mathrm{EZH} 2$ on Th1 and Th2 differentiation are not completely consistent (24-28). Specifically, whereas three investigator groups reported increased IFN- $\gamma$ production from EZH2-deficient T cells cultured under Th1 polarizing conditions (25-27), Tong et al. reported the opposite observation (28). This study found that downregulation of EZH2 decreases the production of IFN- $\gamma$ under Th1 skewing conditions, which is not affected by neutralization of IL- 4 and is not associated with differences in Gata-3 levels, suggesting that EZH2 promotes Th1 differentiation in vitro independently of IL-4 and Gata-3 (28). The authors showed that loss of EZH2 promotes the development of a particular histone methylation signature for genes associated with Th1 differentiation such as Ifng, Tbx21, and Stat4 (28).
These investigators also reported that $\mathrm{EZH} 2$ increases the stability of T-bet (28), suggesting a novel role of EZH2 as an inducer of Th1 differentiation. Interestingly, enhancement of Th1 differentiation through EZH2 was associated with the development of T cellmediated aplastic anemia in transgenic mouse models, suggesting that targeting EZH2 might be a possible therapeutic approach for the treatment of aplastic anemia (28). These results are in agreement with a study by Jacob et al. who found that chromatin binding of the EZH2 to cytokine promoters in $\mathrm{CD}^{+} \mathrm{T}$ cells is associated with induction of Th1 and Th2 cytokines, suggesting a positive role of EZH2 toward cytokine gene transcription in $\mathrm{CD}^{+} \mathrm{T}$ cells (29). However, it is possible that the reason for this discrepancy might be purely methodological. Specifically, it was observed that enhanced Th2 differentiation and increased production of IL- 4 by EZH2-deficient cells opposes Th1 polarization (25-27). Notably, the only study that observed decreased IFN- $\gamma$ production - a hallmark of Th1 polarization - did not report the method of naïve $\mathrm{T}$ cell purification prior to in vitro polarizing culture (28). Therefore, it is possible that different isolation methods and lower purification efficiency might have resulted in the diminished ability of naïve EZH2-deficient T cells to be polarized toward Th1 phenotype observed in this one study.

Although EZH2 is a known methyltransferase acting as epigenetic regulator, Su et al. highlighted a cytosolic function of this protein in mediating $\mathrm{T}$ cell receptor-induced actin polymerization and T cell differentiation (14). Subsequently, Gunawan et al. showed that EZH2 deficiency impairs integrin-dependent 
migration of leukocytes affecting the progression of multiple sclerosis in a mouse autoimmune model further supporting a critical extranuclear function of EZH2 in the development of inflammation and autoimmune disease (30).

\section{EZH2 and Effector T Cells}

Apart from the differentiation of naïve $\mathrm{CD} 4^{+} \mathrm{T}$ cells, the function of the immune system also depends on the survival of differentiated immune cells. Zhang et al. found that EZH2 promotes the survival of differentiated effector $\mathrm{T}$ cells through inhibition of numerous apoptosis pathways, including Fas, TNFR1, DR4, and Mlk1 signaling (25) (Figure 1B). Consistent with this finding, Yang et al. showed that EZH2-deficient mice have decreased survival after intraperitoneal Toxoplasma gondii infection and this was associated with decreased numbers of IFN- $\gamma$ producing $C D 4^{+}$ $T$ cells in the peritoneal exudate (26). These observations suggest that EZH2 is critical for the generation of T effector cell responses in vivo, and the defects observed in $\mathrm{EZH} 2$-deficient mice were attributed to the decreased proliferation of EZH2 effector T cells. The conclusion that EZH2 is critical for the survival, proliferation, and activity of effector T cells was further supported by a study by He et al. who evaluated the role of EZH2 in the development of GVHD using a model of allogeneic bone marrow transplantation (31). Particularly, the authors showed that EZH2 is critical for the survival and proliferation of alloantigen-activated $\mathrm{T}$ cells and the development of GVHD in a major histocompatibility (MHC)mismatched B6 anti-BALB/C mouse model. In that system, IFN- $\gamma$ producing alloreactive $\mathrm{T}$ cells are significantly diminished in the absence of EZH2 (31). Of note, the deletion of EZH2 did not affect the anti-leukemic effect of donor T cells in this mouse model (31). This observation suggested that inhibition of EZH2 might be a reasonable approach to protect patients undergoing bone marrow transplantation from the development of GVHD without compromising the beneficial graft-versus-leukemia effect of allogeneic stem cell transplantation.

\section{EZH2 and T Regulatory Cells}

While the studies presented above supported a critical role of EZH 2 on Th1 and Th2 differentiation of naive $\mathrm{CD}^{+} \mathrm{T}$ cells with very tentative therapeutic implications, the deletion of EZH2 has been associated with significant reduction of FOXP3 (25), suggesting that EZH2 may be critical for the activation of Tregs. FOXP3 co-localizes with EZH2 (32) and it has been shown that the absence of EZH2 is associated with impairment of iTreg differentiation in vitro $(25,27)$. These studies also indicated a possible positive role of EZH2 in the regulation of Treg differentiation, activation, and suppressive function (Figure 1C). Yang et al. evaluated the implication of EZH2 on Treg function and found that CD4-specific deletion of EZH2 in transgenic mice leads to significantly fewer naïve T cells and decreased FOXP3 expression in CD4 cells in the spleen and lymph nodes (26). Interestingly, the authors found that the addition of antibodies against IFN- $\gamma$ and IL-4 reverses the impaired FOXP3 expression in EZH2deficient T cells (26). This observation is consistent with the data of Zhang et al. showing that the FOXP3 reduction due to EZH2 deficiency is reversed in the presence of IFN- $\gamma$ blocking antibody (25). These findings imply that the downregulation of FOXP3 in
EZH2-deficient cells is likely mediated by aberrant production of cytokines, such as IFN- $\gamma$ and IL-4. To expand these findings and understand their biological implications in vivo, Yang et al. studied the functional implications of EZH2 deficiency on the properties of Tregs and determined that EZH2-deficient Tregs failed to protect from the development of autoimmunity in a model of naïve T cell-mediated colitis in vivo (26).

Further highlighting the critical role of EZH2 in the activation of Tregs, DuPage et al. showed that, at the transcription level, EZH2 is the most abundant chromatin modifier induced by CD28 activation in naïve $\mathrm{CD} 4^{+} \mathrm{T}$ cells (33). This is consistent with previous reports supporting that the epigenetic state of Tregs is regulated by pathways related to the $\mathrm{T}$ cell receptor-mediated stimulation $(34,35)$. More importantly, Treg-specific EZH2 deletion renders Tregs phenotypically normal and functional in vitro but unable to properly maintain immune homeostasis in vivo, as supported by the fact that Treg.EZH $2 \Delta / \Delta$ mice presented numerous signs of autoimmunity, including weight and hairy loss and scaly tails (33). The authors showed that EZH2 is responsible for the maintenance of the repressive gene program after Treg activation, which is critical for the recruitment and function of Tregs at the site of inflammation (33). These results support an important role of EZH2 in the activity of Tregs, which may have significant implications not only in the context of autoimmune diseases and cancer.

\section{EZH2 and Regulation of Anti-Tumor Immunity}

Despite the extensive research evaluating the role of EZH2 as a promoter of cancer progression through the induction of cell cycle and inhibition of cancer cell differentiation, the effects of EZH2 expression on the tumor immunity have not been fully appreciated. According to a recent publication by Peng et al., epigenetic alterations of cancer cells by inhibition of EZH2 and DNA methyltransferase 1 (DNMT1) in a mouse model of ovarian cancer resulted in increased expression of the Th1type chemokines CXCL9 and CXCL10 in cancer cells leading to increased trafficking of effector $\mathrm{T}$ cells to the tumor site and decreased tumor volume (36). Conversely, increased expression of EZH2 and DNMT1 in ovarian tumors was associated with decreased infiltration of CD8 ${ }^{+} \mathrm{T}$ cells and worse prognosis (36) (Figure 1D). Furthermore, treatment of ovarian tumors with EZH2 and DNMT1 inhibitors increased the efficacy of tumorassociated antigen-specific $\mathrm{CD}^{+} \mathrm{T}$ cells in response to PD-L1 inhibition. A similar effect of EZH2 was observed in colorectal cancer, where targeting EZH2 in cancer cells augments the expression of CXCL9 and CXCL10 chemokines affecting the infiltration of the tumor by effector $\mathrm{T}$ cells (37). These results support the conclusion that the tumorigenic effects of histone modifications and DNA methylation in cancer cells may be mediated by alteration of immunity in the tumor microenvironment. This conclusion may lead to the introduction of novel therapeutic strategies to improve the efficacy of immunotherapy.

\section{CONCLUSION}

Enhancer of zeste homolog 2 is a methyltransferase, member of the PRC2 complex acting as epigenetic regulator with critical 
implications in maintaining the gene signature particularly associated with cell cycle progression, proliferation, and differentiation. Recent studies suggest that EZH2 has significant implications in carcinogenesis and is a novel target in cancer therapeutics. While EZH2 inhibitors are currently under intense investigation, the implications of EZH2 in the regulation of $\mathrm{T}$ cell differentiation and activity are only now starting to be unraveled. Such effects of EZH2 are of particular interest because targeting T cells-specific $\mathrm{EZH} 2$ functions may have significant implications for the control of autoimmunity, alloreactivity, and anti-tumor immunity. Strikingly, EZH2 appears to function as a crosstalk regulator between cancer and T cells and modulation of EZH2 expression in cancer cells alters their ability to produce Th1-type chemokines and to attract $\mathrm{CD} 8^{+} \mathrm{T}$ effector cells, which mediate anti-tumor function especially in the context of checkpoint blockade immunotherapy. Importantly, EZH2 has activity not only on T effector cells but also on Tregs, rendering the therapeutic targeting of EZH2 a uniquely complex challenge. Further studies will be

\section{REFERENCES}

1. Cao R, Wang L, Wang H, Xia L, Erdjument-Bromage H, Tempst P, et al. Role of histone H3 lysine 27 methylation in polycomb-group silencing. Science (2002) 298:1039-43. doi:10.1126/science.1076997

2. Müller J, Hart CM, Francis NJ, Vargas ML, Sengupta A, Wild B, et al. Histone methyltransferase activity of a Drosophila polycomb group repressor complex. Cell (2002) 111:197-208. doi:10.1016/S0092-8674(02)00976-5

3. Margueron R, Reinberg D. The polycomb complex PRC2 and its mark in life. Nature (2011) 469:343-9. doi:10.1038/nature09784

4. Boyer LA, Plath K, Zeitlinger J, Brambrink T, Medeiros LA, Lee TI, et al. Polycomb complexes repress developmental regulators in murine embryonic stem cells. Nature (2006) 441:349-53. doi:10.1038/nature04733

5. Czermin B, Melfi R, McCabe D, Seitz V, Imhof A, Pirrotta V. Drosophila enhancer of Zeste/ESC complexes have a histone H3 methyltransferase activity that marks chromosomal polycomb sites. Cell (2002) 111:185-96. doi:10.1016/S0092-8674(02)00975-3

6. Schuettengruber B, Chourrout D, Vervoort M, Leblanc B, Cavalli G. Genome regulation by polycomb and trithorax proteins. Cell (2007) 128:735-45. doi:10.1016/j.cell.2007.02.009

7. Hubaux R, Thu KL, Coe BP, MacAulay C, Lam S, Lam WL. EZH2 promotes E2F-driven SCLC tumorigenesis through modulation of apoptosis and cell-cycle regulation. J Thorac Oncol (2013) 8:1102-6. doi:10.1097/ JTO.0b013e318298762f

8. Shen X, Kim W, Fujiwara Y, Simon MD, Liu Y, Mysliwiec MR, et al. Jumonji modulates polycomb activity and self-renewal versus differentiation of stem cells. Cell (2009) 139:1303-14. doi:10.1016/j.cell.2009.12.003

9. Lee TI, Jenner RG, Boyer LA, Guenther MG, Levine SS, Kumar RM, et al. Control of developmental regulators by polycomb in human embryonic stem cells. Cell (2006) 125:301-13. doi:10.1016/j.cell.2006.02.043

10. Bracken AP, Pasini D, Capra M, Prosperini E, Colli E, Helin K. EZH2 is downstream of the $\mathrm{pRB}-\mathrm{E} 2 \mathrm{~F}$ pathway, essential for proliferation and amplified in cancer. EMBO J (2003) 22:5323-35. doi:10.1093/emboj/cdg542

11. Varambally S, Cao Q, Mani RS, Shankar S, Wang X, Ateeq B, et al. Genomic loss of microRNA-101 leads to overexpression of histone methyltransferase EZH2 in cancer. Science (2008) 322:1695-9. doi:10.1126/ science. 1165395

12. Simon JA, Lange CA. Roles of the EZH2 histone methyltransferase in cancer epigenetics. Mutat Res (2008) 647:21-9. doi:10.1016/j. mrfmmm.2008.07.010

13. Kamminga LM, Bystrykh LV, de Boer A, Houwer S, Douma J, Weersing E, et al. The polycomb group gene Ezh2 prevents hematopoietic stem cell exhaustion. Blood (2006) 107:2170-9. doi:10.1182/blood-2005-09-3585 required to clarify the effects of EZH2 on the differentiation, survival, and function of Th, T effector, and Tregs in order to create novel therapeutic strategies in the area of autoimmune diseases, allogeneic transplantation, and cancer.

\section{AUTHOR CONTRIBUTIONS}

TK did the outline of the work and generated the main body of the manuscript and figures; $\mathrm{AC}$ wrote individual subsections of the manuscript; KB wrote subsections of the manuscript; LL provided comments in all sections and wrote additional points; and VB revised the manuscript and figures and provided input in all sections.

\section{FUNDING}

This work was supported by NIH/NCI grants CA183605, CA183605S1, and AI098129-01 and by the DoD grant PC140571.

14. Su IH, Dobenecker MW, Dickinson E, Oser M, Basavaraj A, Marqueron R, et al. Polycomb group protein ezh2 controls actin polymerization and cell signaling. Cell (2005) 121:425-36. doi:10.1016/j.cell.2005.02.029

15. Su IH, Basavaraj A, Krutchinsky AN, Hobert O, Ullrich A, Chait BT, et al. Ezh2 controls B cell development through histone H3 methylation and Igh rearrangement. Nat Immunol (2003) 4:124-31. doi:10.1038/ni876

16. Paul WE, Seder RA. Lymphocyte responses and cytokines. Cell (1994) 76:241-51. doi:10.1016/0092-8674(94)90332-8

17. Zhu J, Paul WE. CD4 T cells: fates, functions, and faults. Blood (2008) 112:1557-69. doi:10.1182/blood-2008-05-078154

18. Mosmann TR, Coffman RL. TH1 and TH2 cells: different patterns of lymphokine secretion lead to different functional properties. Annu Rev Immunol (1989) 7:145-73. doi:10.1146/annurev.iy.07.040189.001045

19. Kuchroo VK, Awasthi A. Emerging new roles of Th17 cells. Eur J Immunol (2012) 42:2211-4. doi:10.1002/eji.201242872

20. Patel DD, Kuchroo VK. Th17 cell pathway in human immunity: lessons from genetics and therapeutic interventions. Immunity (2015) 43:1040-51. doi:10.1016/j.immuni.2015.12.003

21. Zhu J, Paul WE. Heterogeneity and plasticity of T helper cells. Cell Res (2010) 20:4-12. doi:10.1038/cr.2009.138

22. Bettelli E, Korn T, Kuchroo VK. Th17: the third member of the effector T cell trilogy. Curr Opin Immunol (2007) 19:652-7. doi:10.1016/j.coi.2007.07.020

23. Li L, Boussiotis VA. Molecular and functional heterogeneity of T regulatory cells. Clin Immunol (2011) 141:244-52. doi:10.1016/j.clim.2011.08.011

24. Raaphorst FM, Otte AP, van Kemenade FJ, Blokzijl T, Fieret E, Hamer $\mathrm{KM}$, et al. Distinct BMI-1 and EZH2 expression patterns in thymocytes and mature $\mathrm{T}$ cells suggest a role for polycomb genes in human $\mathrm{T}$ cell differentiation. J Immunol (2001) 166:5925-34. doi:10.4049/ jimmunol.166.10.5925

25. Zhang Y, Kinkel S, Maksimovic J, Bandala-Sanchez E, Tanzer MC, Naselli G, et al. The polycomb repressive complex 2 governs life and death of peripheral T cells. Blood (2014) 124:737-49. doi:10.1182/blood-2013-12-544106

26. Yang XP, Jiang K, Hirahara K, Vahedi G, Afzali B, Sciume G, et al. EZH2 is crucial for both differentiation of regulatory $\mathrm{T}$ cells and $\mathrm{T}$ effector cell expansion. Sci Rep (2015) 5:10643. doi:10.1038/srep10643

27. Tumes DJ, Onodera A, Suzuki A, Shinoda K, Endo Y, Iwamura C, et al. The polycomb protein Ezh2 regulates differentiation and plasticity of CD4(+) T helper type 1 and type 2 cells. Immunity (2013) 39:819-32. doi:10.1016/j. immuni.2013.09.012

28. Tong Q, He S, Xie F, Mochizuki K, Liu Y, Mochizuki I, et al. Ezh2 regulates transcriptional and posttranslational expression of T-bet and promotes Th1 cell responses mediating aplastic anemia in mice. J Immunol (2014) 192:5012-22. doi:10.4049/jimmunol.1302943 
29. Jacob E, Hod-Dvorai R, Schif-Zuck S, Avni O. Unconventional association of the polycomb group proteins with cytokine genes in differentiated $\mathrm{T}$ helper cells. J Biol Chem (2008) 283:13471-81. doi:10.1074/jbc.M709886200

30. Gunawan M, Venkatesan N, Loh JT, Wong JF, Berger H, Neo WH, et al. The methyltransferase Ezh2 controls cell adhesion and migration through direct methylation of the extranuclear regulatory protein talin. Nat Immunol (2015) 16:505-16. doi:10.1038/ni.3125

31. He S, Xie F, Liu Y, Tong Q, Mochizuki K, Lapinski PE, et al. The histone methyltransferase Ezh2 is a crucial epigenetic regulator of allogeneic T-cell responses mediating graft-versus-host disease. Blood (2013) 122:4119-28. doi:10.1182/blood-2013-05-505180

32. Arvey A, van der Veeken J, Samstein RM, Feng Y, Stamatoyannopoulos JA, Rudensky AY. Inflammation-induced repression of chromatin bound by the transcription factor Foxp3 in regulatory T cells. Nat Immunol (2014) 15:580-7. doi:10.1038/ni.2868

33. DuPage M, Chopra G, Quiros J, Rosenthal WL, Morar MM, Holohan D, et al. The chromatin-modifying enzyme Ezh2 is critical for the maintenance of regulatory $\mathrm{T}$ cell identity after activation. Immunity (2015) 42:227-38. doi:10.1016/j.immuni.2015.01.007

34. Ohkura N, Hamaguchi M, Morikawa H, Sugimura K, Tanaka A, Ito Y, et al. $\mathrm{T}$ cell receptor stimulation-induced epigenetic changes and Foxp3 expression are independent and complementary events required for Treg cell development. Immunity (2012) 37:785-99. doi:10.1016/j.immuni.2012.09.010
35. Samstein RM, Arvey A, Josefowicz SZ, Peng X, Reynolds A, Sandstrom $\mathrm{R}$, et al. Foxp3 exploits a pre-existent enhancer landscape for regulatory $\mathrm{T}$ cell lineage specification. Cell (2012) 151:153-66. doi:10.1016/j. cell.2012.06.053

36. Peng D, Kryczek I, Nagarsheth N, Zhao L, Wei S, Wang W, et al. Epigenetic silencing of TH1-type chemokines shapes tumour immunity and immunotherapy. Nature (2015) 527:249-53. doi:10.1038/nature15520

37. Nagarsheth N, Peng D, Kryczek I, Wu K, Li W, Zhao E, et al. PRC2 epigenetically silences Th1-type chemokines to suppress effector T cell trafficking in colon cancer. Cancer Res (2016) 76(2):275-82. doi:10.1158/0008-5472. CAN-15-1938

Conflict of Interest Statement: The authors declare that the research was conducted in the absence of any commercial or financial relationships that could be construed as a potential conflict of interest.

Copyright (c) 2016 Karantanos, Christofides, Bardhan, Li and Boussiotis. This is an open-access article distributed under the terms of the Creative Commons Attribution License (CC BY). The use, distribution or reproduction in other forums is permitted, provided the original author(s) or licensor are credited and that the original publication in this journal is cited, in accordance with accepted academic practice. No use, distribution or reproduction is permitted which does not comply with these terms. 